\title{
South African Tick Bite Fever: An Overview
}

\author{
John Frean ${ }^{\mathrm{a}, \mathrm{b}}$ Wayne Grayson ${ }^{\mathrm{b}, \mathrm{c}}$ \\ aParasitology Reference Laboratory, Centre for Emerging Zoonotic and Parasitic Diseases, \\ National Institute for Communicable Diseases, Johannesburg, South Africa; ${ }^{\text {bS }}$ Chool of \\ Pathology, Faculty of Health Sciences, University of the Witwatersrand, Johannesburg, \\ South Africa; 'Ampath National Laboratories, Johannesburg, South Africa
}

\section{Keywords}

South African tick bite fever $\cdot$ Rickettsia conorii $\cdot$ Rickettsia africae

\section{Abstract}

The rickettsiae are a diverse group of vector-borne zoonotic bacterial pathogens. The two common spotted fever diseases in existence in southern Africa are boutonneuse fever-like tick bite fever (TBF), caused by Rickettsia conorii, and African TBF, caused by $R$. africae. This review addresses demographic, epidemiological, clinical, diagnostic, therapeutic, and preventive aspects of TBF in the southern African context, including a discussion of the dermatopathological findings and potential diagnostic pitfalls.

(C) 2019 The Author(s)

Published by S. Karger AG, Basel

\section{Introduction}

The rickettsiae comprise a diverse group of vector-borne zoonotic bacterial pathogens, whose clinical diseases can be usefully classified into two groups: typhus (louse- or fleaborne) and spotted fevers, which include South African tick bite fever (TBF). There are two common spotted fever diseases of southern Africa: boutonneuse fever-like TBF (BFL-TBF, caused by Rickettsia conorii) and African TBF (ATBF, caused by R. africae) [1]. Single cases of other spotted fever rickettsial infections, namely, $R$. aeschlimannii and $R$. mongolotimonae, have also been described in South Africa, but their contribution to the local burden of disease is not known, as molecular diagnosis and genotyping are not routinely used [2,3]. 
R. conorii is usually transmitted by dog and kennel ticks (Haemaphysalis elliptica and Rhipicephalus simus) in periurban or peridomestic situations. In contrast, $R$. africae is typically transmitted by particular cattle and game ticks (Amblyomma hebraeum in southern Africa) in rural settings. Surveys have shown up to $70 \%$ seroprevalence of ATBF in subSaharan areas where Amblyomma ticks and cattle farming coincide [4]. Despite this, there are very few clinical case reports of TBF in indigenous populations [5], presumably because of mild or inapparent cases of disease.

In Zimbabwe, annual case incidence rates of ATBF have been estimated as 60-80 per 10,000 patients [6]. A recent aetiological study of non-malarial acute febrile illness in a rural cattle-herding community in Mpumalanga Province, South Africa, showed serological evidence (IgM antibodies) of recent TBF in $24.5 \%$ of patients with a febrile illness; $63.4 \%$ had IgG antibodies. In the same community, $92.2 \%$ of a healthy control group were seropositive, showing that exposure to TBF rickettsiae is common [7]. TBF is commonly recognised in nonAfrican patients in South Africa, but the incidence is not known. Tourists and travellers have been the subjects of case series publications [8]. The incidence rates of infection have been estimated to be $4-5 \%$ in visitors from Europe, which are higher than those for other febrile illnesses such as malaria and typhoid fever. There is a large population at risk, for instance, game reserve visitors, hunters, soldiers, and farmers [8].

\section{Review/Discussion}

\section{Clinical Features}

TBF is common in South Africa, although recognised cases are probably far outnumbered by subclinical ones. Larval and nymph-stage ticks typically transmit the diseases; larvae ("pepper ticks") are often unnoticed because they are very small. $R$. conorii infections (BFLTBF) begin, after an incubation period of 5-7 days, with a consistent prodrome of malaise, fever, headache, nightmares, and myalgia. An eschar is the primary lesion and marks the site of attachment of the infected tick; it consists of a central necrotic area surrounded by inflamed skin (Fig. 1). The eschar is not always obvious; it may be under scalp hair, behind the ear, in the anogenital area, or in other cryptic body sites. About 3 days after the onset of symptoms, a generalised coarse maculopapular rash appears (Fig. 2); its distribution typically includes the palms and soles.

The clinical presentation varies from very mild to severe and even fatal disease, the latter particularly, but not exclusively, amongst elderly or debilitated people [9]. The capacity of BFL-TBF to cause severe disease was recognised many years ago $[10,11]$, and even at present, a few deaths from severe TBF are reported each year in South Africa [12]. Complications include encephalitis, confusion, or coma; pneumonia; pulmonary embolism following deep vein thrombosis; bleeding; gangrene; hepatorenal failure; and myocarditis. Especially if diagnosis and treatment are delayed, BFL-TBF cases can present with multiorgan involvement, and mimic meningococcal septicaemia, other fulminant gram-negative septicaemia with disseminated intravascular coagulopathy, or viral haemorrhagic fever such as CrimeanCongo haemorrhagic fever.

ATBF ( $R$ a africae) tends to be a milder disease without life-threatening complications, although occasional CNS neuropathy and myocarditis have been reported [4]. The ATBF prodrome is similar to that of $R$. conorii infection; characteristic, but not consistent, distinguishing features are multiple eschars, tender regional lymphadenopathy, rashless illness, or only scattered and/or vesicular rash elements [8]. 
Fig. 1. Clinical image of a typical eschar at the site of a tick bite.
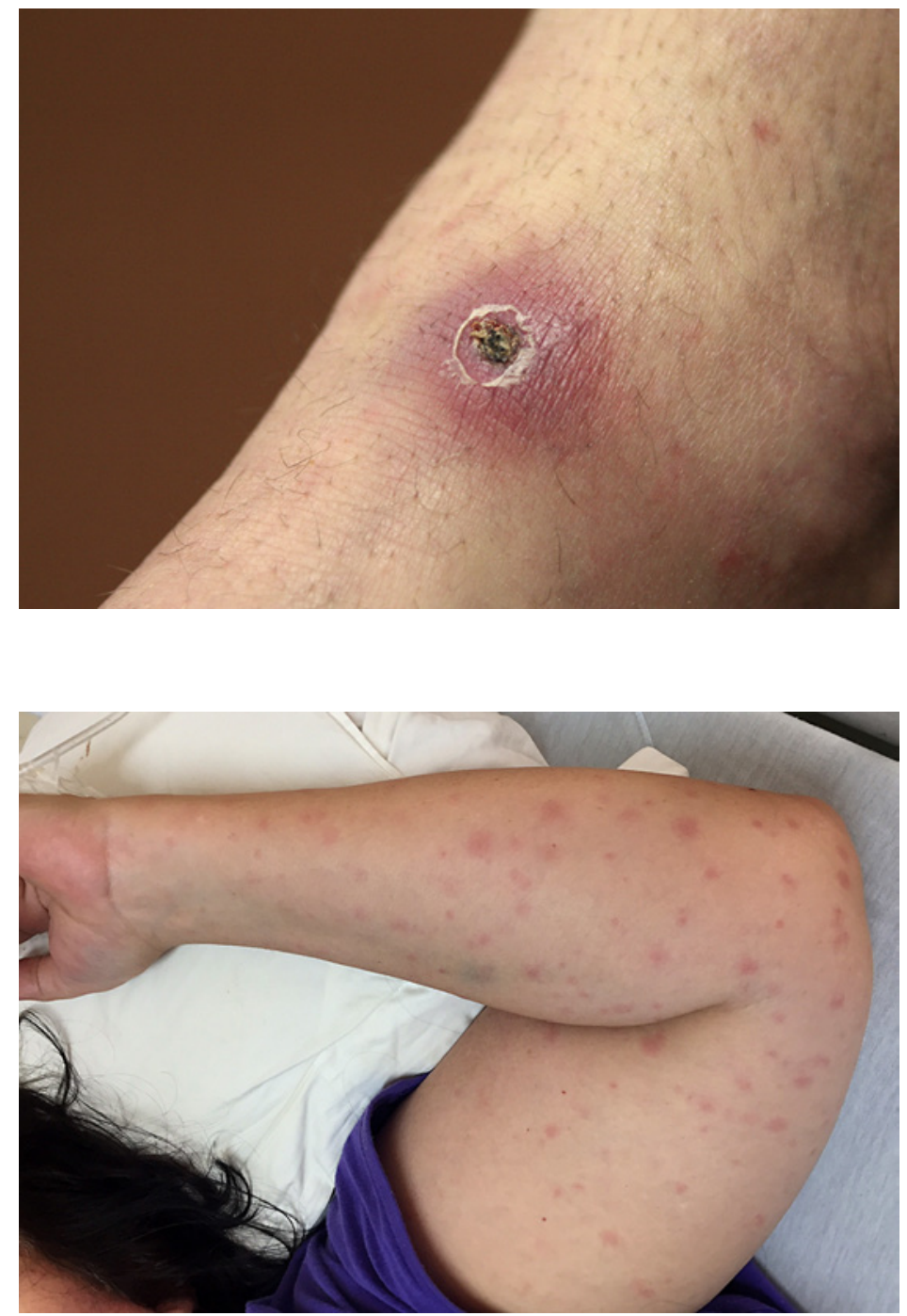

Fig. 2. Clinical image of a maculopapular skin rash associated with Rickettsia conorii infection.

\section{Histopathology}

Biopsies from the eschar show a wedge-shaped zone of coagulative necrosis involving the epidermis and superficial dermis (Fig. 3). Blood vessels near the apex of the necrotic zone often exhibit features of a necrotising vasculitis, sometimes accompanied by vascular thrombosis (Fig. 4) [13-15]. A surrounding dermal infiltrate of lymphocytes and macrophages is not uncommon. Skin biopsies representative of the maculopapular rash reveal a variable superficial and often deeper perivascular lymphocytic infiltrate (Fig. 5). There are accompanying stigmata of a lymphocytic vasculopathic reaction, with endothelial swelling, fibrinoid change in the involved vessel walls, and perivascular erythrocytic extravasation (Fig. 6, 7); small vessel thrombi may also be observed $[13,16]$. Rarely, dyskeratotic keratinocytes or foci of spongiosis can be seen (pers. observation); these latter changes, however, may also be encountered in the context of a viral exanthem or morbilliform drug rash, emphasising the need for careful clinicopathological correlation prior to rendering a definitive diagnosis. In addition to all important serological studies, the diagnosis may also be confirmed by PCR, including PCR performed on samples obtained from eschar swabs $[17,18]$. 
Fig. 3. Low-power view of a tick bite eschar. Note the inflammatory exudate overlying a zone of epidermal and superficial dermal necrosis, with a surrounding vital response.

Fig. 4. Medium-power view of a tick bite eschar. Vasculitis and vascular thrombosis are evident below the base of the necrotic lesion, along with a background lymphohistiocytic and neutrophilic inflammatory infiltrate.
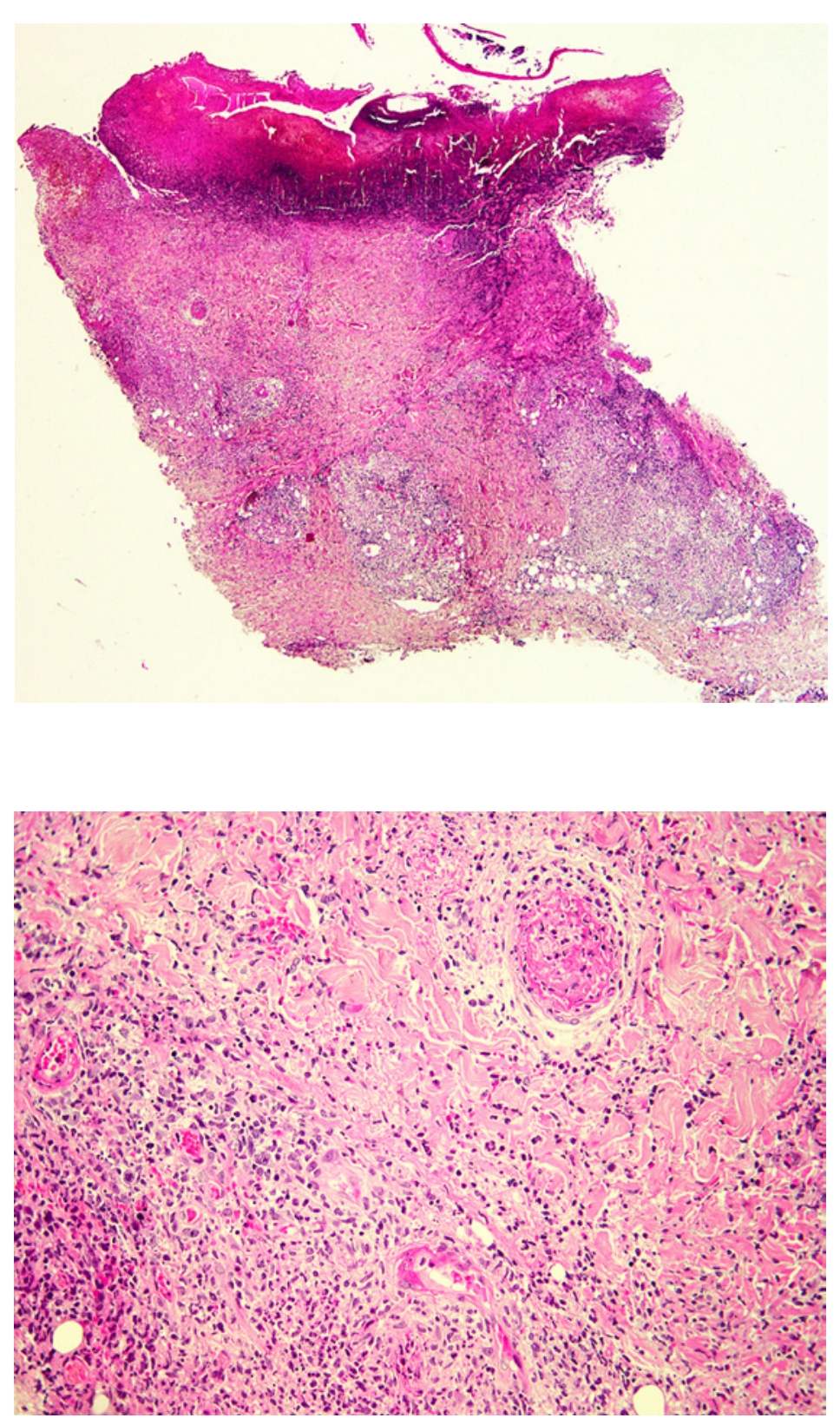

\section{Diagnostic Issues}

The triad of fever, eschar, and rash occurs in $50-75 \%$ of cases of TBF, but there are less typical presentations. The eschar may resemble an infected insect bite or other skin trauma. The rash may suggest rubella, measles, secondary syphilis, disseminated gonococcal disease, enterovirus or arbovirus infections, leptospirosis, typhoid, immune complex vasculitis, or drug reactions. Meningococcal rashes can look similar, but the onset and progression of the illness is much faster than with TBF. Malaria is an important differential diagnosis of the nonspecific prodrome in travellers. Early serological tests are often negative and repeat testing is required; treatment should not be delayed solely because of negative antibody tests. Early treatment may abort seroconversion. Specific microimmunofluorescence is the serological method of choice, but it will not distinguish between $R$. conorii and $R$. africae infections. The 
Fig. 5. South African tick bite fever due to $R$. conorii infection. This low-power view shows a superficial and deep perivascular dermal lymphocytic infiltrate.
Fig. 6. South African tick bite fever due to $R$. conorii infection. A lymphocytic vasculopathic reaction is observed in this highpower photomicrograph.
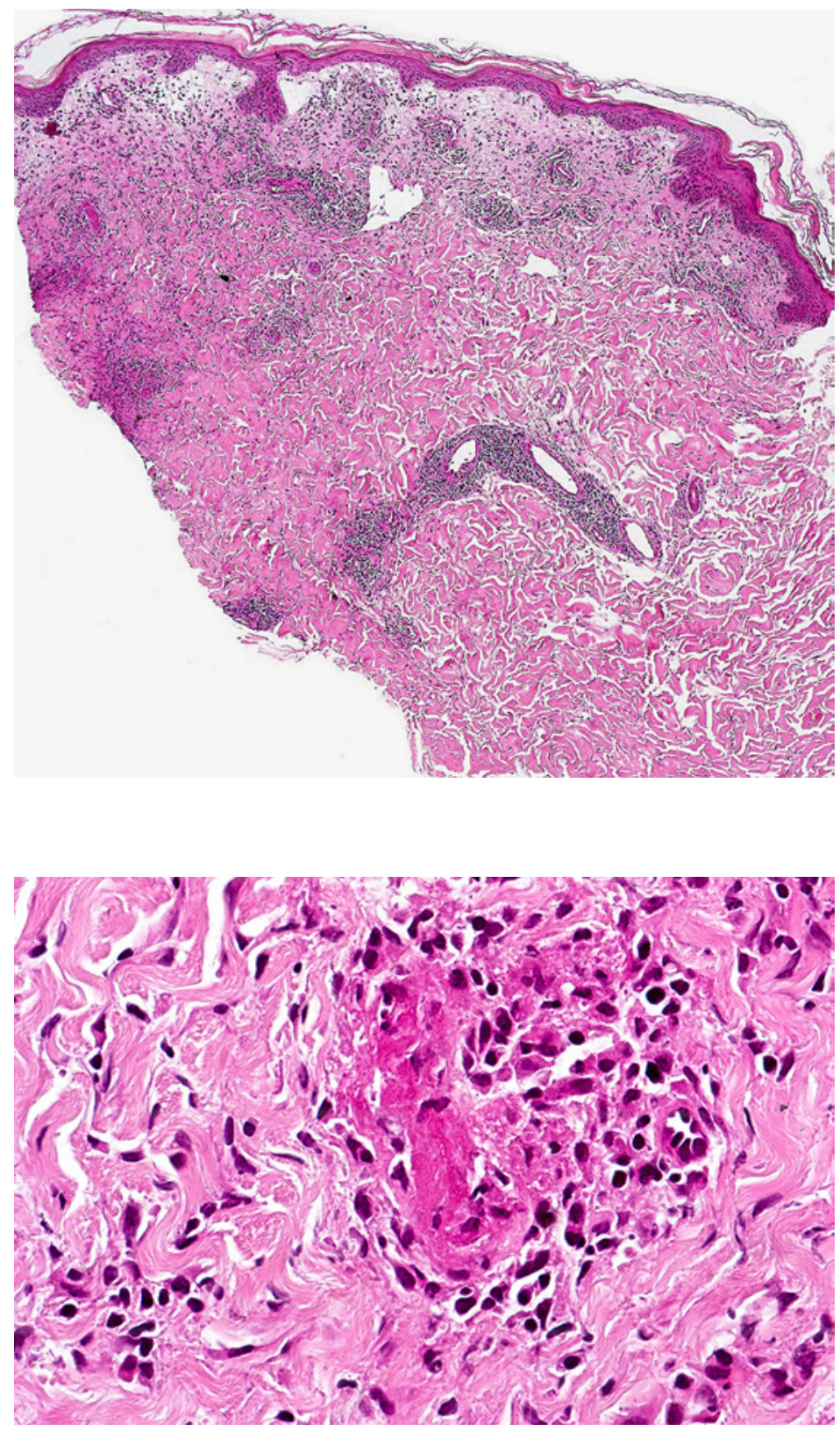

Weil-Felix agglutination test is now regarded as obsolete. PCR has proved to be a useful diagnostic test in some cases where there is continuing active vasculitis, but the yield on venous blood is lower than on dry cotton wool swabs of the eschar, which can give positive results even after treatment has started. As with serological investigations, treatment should not be withheld because of negative PCR results. In most patients the white blood cell count remains within the normal range. In severe cases neutropenia and thrombocytopenia occur, along with biochemical markers of multiorgan involvement.

\section{Treatment and Prevention}

Many infections are mild, but TBF can be very severe and therapeutic delay should be avoided. Doxycycline is the treatment of choice. For adults, doxycycline $100 \mathrm{mg}$ twice daily 
Fig. 7. High-power photomicrograph of a skin biopsy from a proven case of South African tick bite fever due to $R$. conorii. There is conspicuous perivascular erythrocytic extravasation in the upper dermis in this example.

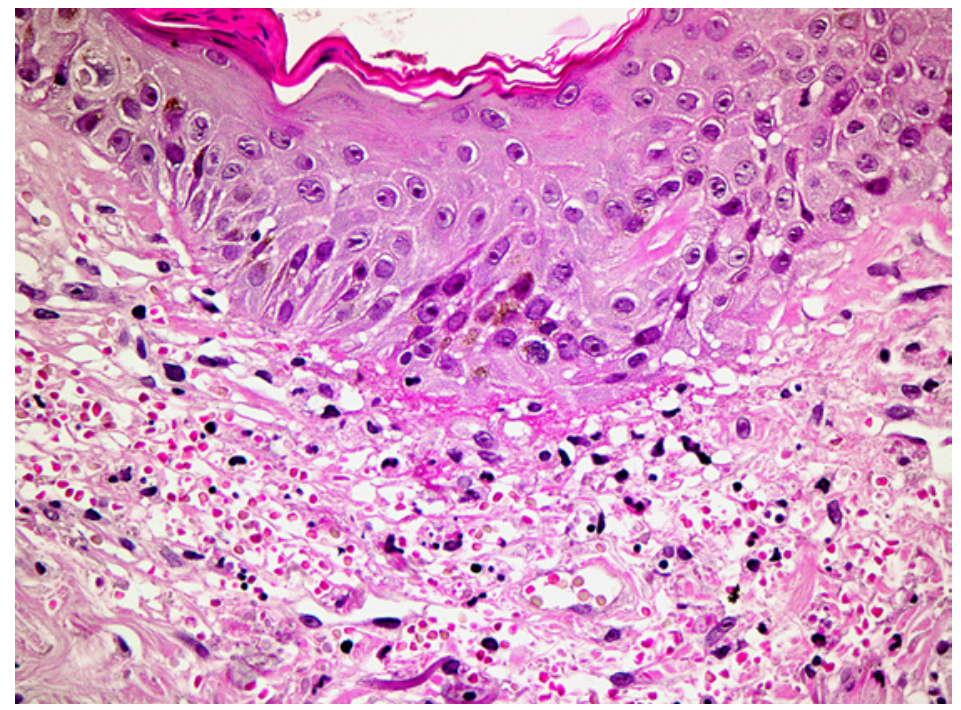

for 5-7 days is recommended, although shorter courses may be adequate [19]. Doxycycline is highly effective, and if there is no clinical response within $48 \mathrm{~h}$, the possibility of another diagnosis should be considered. A fluoroquinolone such as ciprofloxacin may be the only available option for critically ill patients unable to tolerate oral medication, as parenteral tetracycline is unavailable in South Africa. Previously, chloramphenicol was used in this situation, but fluoroquinolones are safer. Erythromycin has poor efficacy [20], and there are few clinical data to recommend new macrolides such as clarithromycin and azithromycin, although, like fluoroquinolones, they may have a place in supplementing initial doxycycline treatment and in covering other possible bacterial pathogens.

As severe TBF can be life-threatening in patients of any age group, initial treatment with the most effective agent, doxycycline, should be considered for all patients with more than the mildest symptoms. For children less than 8 years of age and pregnant women in this category, an initial day or two of doxycycline treatment should be followed by 3-5 days of a macrolide. Ticks are repelled by DEET ( $N, N$-diethyl- $m$-toluamide)-containing products, but these have a limited duration of activity and need to be reapplied periodically. Careful examination for attached ticks should be done after having walked in tick-infested vegetation, remembering that larval ticks are extremely small (1-2 $\mathrm{mm}$ in diameter). Ticks need to stay attached for 24-48 h before they transmit the infection, so a thorough daily skin check and removal is an effective preventive measure. Pre- or post-tick bite antibiotic prophylaxis is reputed to be useless, since it merely prolongs the incubation period. Dogs should be treated with suitable long-acting topical ectoparasiticides (e.g., pyriprole). Attached ticks on pets should be removed and disposed of carefully - TBF has been acquired via the conjunctiva by tick contents splashed in the eye when ticks are crushed [21].

\section{Acknowledgements}

Dr. Tirelo Pitjadi, Ampath National Laboratories, Johannesburg, South Africa, is thanked for the histological sections used for Figures 3 and 4. Figures 5 and 6 were kindly provided by Prof. Phillip H. McKee, Boussais, France. 


\section{Statement of Ethics}

The authors have no ethical conflicts to disclose.

\section{Disclosure Statement}

The authors have no conflicts of interest to declare.

\section{Author Contributions}

J.F. compiled the Introduction and the sections addressing clinical features, diagnostic issues, and treatment and prevention. W.G. compiled the Abstract and histopathology sections, and was also responsible for the photomicrographs and general formatting and editing of the manuscript.

\section{References}

1 Kelly P, Matthewman L, Beati L, Raoult D, Mason P, Dreary M, et al. African tick-bite fever: a new spotted fever group rickettsiosis under an old name. Lancet. 1992 0ct;340(8825):982-3.

2 Pretorius AM, Birtles RJ. Rickettsia mongolotimonae infection in South Africa. Emerg Infect Dis. 2004 Jan; 10(1):125-6.

3 Pretorius AM, Birtles RJ. Rickettsia aeschlimannii: a new pathogenic spotted fever group rickettsia, South Africa. Emerg Infect Dis. 2002 Aug;8(8):874.

4 Jensenius M, Fournier PE, Kelly P, Myrvang B, Raoult D. African tick bite fever. Lancet Infect Dis. 2003 Sep;3(9): $557-64$.

5 Cohen GL, Blumberg LS, Karstaedt AS. Tick bite fever in black South Africans-a rare disease? J Infect. 1996 May;32(3):235-7.

6 Kelly PJ, Mason PR, Matthewman LA, Raoult D. Seroepidemiology of spotted fever group rickettsial infections in humans in Zimbabwe. J Trop Med Hyg. 1991 Oct;94(5):304-9.

7 Simpson GJ, Quan V, Frean J, Knobel DL, Rossouw J, Weyer J, et al. Prevalence of selected zoonotic diseases and risk factors at a human-wildlife-livestock interface in Mpumalanga Province, South Africa. Vector Borne Zoonotic Dis. 2018 Jun;18(6):303-10.

8 Jensenius M, Fournier PE, Vene S, Hoel T, Hasle G, Henriksen AZ, et al.; Norwegian African Tick Bite Fever Study Group. African tick bite fever in travelers to rural sub-Equatorial Africa. Clin Infect Dis. 2003 Jun;36(11):1411-7.

9 Loubser MD, Davies VA, Meyers KE, Christianson AL. Severe illness caused by Rickettsia conorii. Ann Trop Paediatr. 1993;13(3):277-80.

10 Gear J. Complications in tick-bite fever. A survey of fifty cases. S Afr Med J. 1939;13(1):35-6.

11 Gear JH, Miller GB, Martins H, Swanepoel R, Wolstenholme B, Coppin A. Tick-bite fever in South Africa. The occurrence of severe cases on the Witwatersrand. S Afr Med J. 1983 May;63(21):807-10.

12 Fatal tick bite fever, Western Cape Province. NICD Communicable Diseases Communiqué. 2018;17(1):2-3.

13 Grayson W. Infectious diseases of the skin. In: Calonje EJ, Brenn T, Lazar A, Billings S, editors. McKee's pathology of the skin with clinical correlations. 5th ed. Philadelphia: Elsevier; 2018.

14 Tsai YS, Wu YH, Kao PT, Lin YC. African tick bite fever. J Formos Med Assoc. 2008 Jan;107(1):73-6.

15 Kim J, Smith KJ, Naefie R, Skelton H. Histopathologic features of and lymphoid populations in the skin of patients with the spotted fever group of rickettsiae: southern Africa. Int J Dermatol. 2004 Mar;43(3):188-94. Carlson JA, Chen KR. Cutaneous vasculitis update: neutrophilic muscular vessel and eosinophilic, granulomatous, and lymphocytic vasculitis syndromes. Am J Dermatopathol. 2007 Feb;29(1):32-43.

17 Parola P, Paddock CD, Socolovschi C, Labruna MB, Mediannikov O, Kernif T, et al. Update on tick-borne rickettsioses around the world: a geographic approach. Clin Microbiol Rev. 2013 Oct;26(4):657-702.

18 Delord M, Socolovschi C, Parola P. Rickettsioses and Q fever in travelers (2004-2013). Travel Med Infect Dis. 2014 Sep-Oct;12(5):443-58.

19 Raoult D, Drancourt M. Antimicrobial therapy of rickettsial diseases. Antimicrob Agents Chemother. 1991 Dec; 35(12):2457-62.

20 Miller GB, Gear JH. Treatment of tick-bite fever with erythromycin. S Afr Med J. 1984 Nov;66(18):694-7.

21 Gear JH. Tick typhus. In: Gear JH, editor. Handbook of viral and rickettsial hemorrhagic fevers. Boca Raton: CRC Press; 1988. p. 217. 